\title{
¿DONDE SITUAR LOS ESFUERZOS DE MEJORA?: POLÍTICA EDUCATIVA, ESCUELA Y AULA
}

Antonio Bolívar ${ }^{*}$

\begin{abstract}
RESUMEN: El artículo hace una revisión de las teorías actuales del cambio educativo, en particular del papel del establecimiento escolar como una unidad base de la acción educativa y de la mejora. Se da cuenta del "viaje" recorrido en la búsqueda de la mejora: de las políticas centralizadas externas a situar la escuela como unidad estratégica y, en la última década, convertir el incremento del aprendizaje de los alumnos en el espacio privilegiado. De acuerdo con las lecciones aprendidas, un nuevo paradigma de la política educativa y de la innovación aboga por un equilibrio entre las presiones externas que estimulen la mejora con la necesaria autonomía escolar, donde el aprendizaje de todos los alumnos se constituyen en el foco del cambio. A su servicio se subordinan los cambios curriculares, organizativos o apoyos de la política educativa que puedan promoverlo.
\end{abstract}

Palabras clave: Mejora escolar. Políticas educativas. Establecimiento educativo. Buena enseñanza y aprendizaje.

\section{WHERE SHOULD WE FOCUS OUR IMPROVEMENT EFFORTS? EDUCATIONAL POLICIES, SCHOOLS AND CLASSROOMS}

ABSTRACT: This paper reviews the current theories on educational changes, specifically the role of educational establishments as basic units of educational action and improvement. It accounts for the ground covered in search of this improvement: from external centralized policies that make school into a strategic unit to, in the past decade, highlighting the increase in student learning as a privileged topic. Based on the lessons learned, a new paradigm of educational policy and innovation argues for a balance between the external pressures that stimulate improvement and the necessary school au-

Facultad de Ciencias de la Educación, Departamento de Didáctica y Organización Escolar de la Universidad de Granada (España).E-mail: abolivar@ugr.es

Educ. Soc., Campinas, vol. 26, n. 92, p. 859-888, Especial - Out. 2005 
tonomy, where the learning of all of the students becomes the focus of changes. Bearing this in mind, we look into curricular and organizational changes or supports for educational policies that can promote such changes.

Key words: School improvement. Educational policies. Educational establishments. Powerful teaching and learning

\section{Introducción}

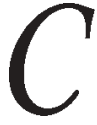

ontamos con un conocimiento acumulado para revisar y extraer lecciones de los resultados (exitosos o fracasados) que las diferentes estrategias han tenido en incrementar el aprendizaje de los alumnos, el desarrollo profesional y organizativo. Los últimos treinta años, pródigo en reformas en los distintos países, han adoptado "geografías sociales" diferenciales de provocar la mejora (Hargreaves, 2002). Ha sido común distinguir, particularmente desde los modelos de investigación sobre eficacia escolar, diferentes niveles en la organización de la educación: el alumnado, profesorado y aula (classroom level), la escuela como unidad (school level), y el contexto de política educativa (policy level).

En este artículo vamos a enmarcar lo que hemos aprendido sobre dónde situar las estrategias privilegiadas de mejora según ámbitos de decisión e intervención: si estimular las dinámicas internas de las escuelas, por los impulsos e imposiciones externas de la política, o - en último extremo - todo se juega en la práctica docente en el aula. Sucesivos vaivenes, que en las reformas norteamericanas - comenta Elmore (1997) - más se asemejan a una opereta o teatro del absurdo, han llevado de primar las acciones externas de la política a estimular las dinámicas internas de los establecimientos escolares por implicación (compromiso) o por presión (evaluación o estándares), para, en último extremo, pensar que todo debe dirigirse a las condiciones que mejoran directamente la práctica docente para todos los alumnos.

Un cierto desencanto sobre las posibilidades que las reformas, empaquetadas externamente, han tenido para transformar la realidad educativa en el último tercio del siglo pasado, ha motivado volver la mirada a tomar a la escuela como lugar estratégico de un cambio generado desde abajo. Si el establecimiento educativo como organización al hilo de movimientos como "escuelas eficaces" o "mejora de la escue- 
la"- se constituyó en los ochenta como unidad básica de cambio, dejando el trabajo en el aula en un segundo plano, dependiente del conjunto; actualmente - en una cierta vuelta -, volvemos a reivindicar el lugar clave del aula y los procesos de enseñanza y aprendizaje. Queremos dar cuenta de qué ha pasado por medio en este viaje, para, mirando hacia atrás, volver ahora a lo que siempre pareció obvio y que algunas "olas" han hecho, si no olvidar, sí silenciar indebidamente. En último extremo todo se jugará en que haya un programa de enseñanza coherente, que conjugue en una misma dirección los aspectos curriculares y didácticos, las condiciones de trabajos y los recursos (Newman, Smith y otros, 2001).

No obstante, a nivel internacional se está pensando si no hemos distraído la atención a lo que debía ser el foco de cualquier política curricular: cómo garantizar una buena enseñanza para todos los alumnos. Hopkins (1998, p. 1049) habla explícitamente del olvido de este nivel ("the missing instruccional level"). Por eso, nos estamos replanteando el papel de la escuela como organización y volviendo, en parte, a situar el nivel del aula en nuestro núcleo de preocupación, para lograr un buen aprendizaje para todos. De hecho, en toda la investigación educativa occidental hay una tendencia creciente a concentrarse en "variables próximas" a la mejora de la enseñanza, en lugar de "variables distantes". La conclusión es si después de habernos concentrado en que los cambios educativos debían dirigirse a nivel organizativo o de escuela, la presión actual por los resultados y la libre concurrencia entre las escuelas por conseguir alumnos está llevando a poner en primer plano que deben afectar directamente al incremento del aprendizaje de los alumnos (student achievement).

Hemos expuesto en otro lugar (Bolívar, 2004) algunas razones por las que la escuela como organización se constituyó en los ochenta como unidad básica de cambio, quedando en gran medida el aprendizaje en el aula en un segundo plano, dependiente del conjunto. A mediados de los noventa, con la presión por los resultados de aprendizaje, en una cierta vuelta, la mejora de los procesos de enseñanza y aprendizaje se erigen en el núcleo de cualquier propuesta de cambio. En cualquier caso, en esta puesta en primer plano de la acción docente en el aula, se recogen las lecciones aprendidas a nivel de escuela, por lo que el aula aparece ahora anidada en otros muchos entornos, procesos y relaciones. Y, por tanto, si el blanco central (target) es el aprendizaje y rendi- 
¿Donde situar los esfuerzos de mejora?: política educativa, escuela y aula

miento del alumno, para mejorarlo hay que actuar paralelamente en los otros. Lo expresa bien Laura Stoll (2002, p. 27), con estas palabras:

Es absolutamente necesario situar los esfuerzos tanto en la dimensión de aula como en las dimensiones globales de la escuela. Sin poner el interés en ambos aspectos es muy difícil la mejora. Los mensajes de la investigación sobre la eficacia de la escuela dejan patente que lo que sucede en las aulas marca las diferencias en el aprendizaje y en el progreso de los alumnos y alumnas. Pero también sabemos que sin el apoyo institucional que permita a los profesores observarse mutuamente y poner en práctica estrategias aprendidas tanto en cursos externos como en procesos de investigaciónacción, las posibilidades de éxito son limitadas. Las estructuras y la organización facilitan los procesos de cambio y mejora.

\section{Una mirada al cambio educativo}

Las políticas de mejora de la educación de las últimas décadas han recorrido diversas "olas", con incidencia y tempo variables según los países. Tras las estrategias de arriba-abajo con los gobiernos conservadores de los ochenta, una "segunda ola" (reestructuración) se dirigió a rediseñar la organización de las escuelas y el ejercicio de la profesión docente. Actualmente, en una especie de "tercera ola", se da un paso más en el rediseño organizativo, para poner el foco en el aprendizaje de los alumnos y en el rendimiento de la escuela, sin el cual no cabe hablar de mejora o calidad. Cambiar el núcleo de la enseñanza (la "core technology") supone, entre otras cosas, reconocer que un profesorado capacitado es un factor crítico para una mejor educación, crear un entorno donde el aprendizaje sobre la enseñanza sea parte del propio ejercicio profesional, desarrollar buenas visiones de la enseñanza y un conjunto de estándares a conseguir, incentivando un rendimiento de cuentas orientado a la mejora.

En una primera mirada vertical y en un plano general, en efecto, podríamos afirmar que el lugar de la mejora ha ido paulatinamente girando - en la referida "primera ola" - de la iniciativa de la administración política al establecimiento educativo como unidad de cambio y, más recientemente, al aula como espacio privilegiado de intervención educativa. Más internamente (de dentro a fuera), el asunto es más complejo porque, desde un modelo que mantenga una dinámica entre niveles, el 
buen hacer docente en el aula no se mantiene sin una coherencia horizontal acorde en la escuela, y éste sin una política que incite y apoye. Lo que sucede internamente (inside, los que trabajan en el centro) precisa salir fuera (insidelout) y, a la vez, las presiones de fuera deben potenciar lo de dentro (outsidelin), en un cierto menage à trois (Fullan, 2000b). Si las reformas externas quieren tener algún impacto deben dirigirse a potenciar la capacidad interna de los establecimientos escolares para llevarla a cabo, y ésta debe tener un impacto en los aprendizajes de los alumnos. De este modo, una de las principales cuestiones es reimaginar nuevos caminos que conecten más decididamente la dimensión estructural (organizativa y curricular) con la propiamente pedagógica.

Después de varias décadas dedicadas a introducir cambios en el currículum y de analizar e investigar la vida de tales innovaciones, hemos aprendido que es un proceso complejo, no dependiente sólo de voluntades individuales ni de alteraciones estructurales, que debe implicar a los miembros en dinámicas de trabajo y compromisos que capaciten al centro para autorrenovarse, con la esperanza de que puedan institucionalizarse, formando entonces parte de la cultura organizativa del centro escolar (Fullan, 2002b). Como ha mantenido Gather Thurler (2001), el establecimiento escolar juega necesariamente un relevante papel en la construcción del sentido del cambio, porque constituye el entorno del trabajo cotidiano, la comunidad de pertenencia y el contexto de la educación. No obstante, hay ciertas culturas y modos de funcionar las escuelas que son más propicias a la innovación, por lo que las estrategias de mejora se deben dirigir a crear aquellas condiciones que sabemos la favorecen.

La teoría de la innovación - podríamos decir ahora que ha finalizado el siglo - ha sido el último intento moderno por controlar, gestionando de un modo racionalista, los procesos de cambio educativo, al calor del progreso socioeconómico. De hecho, las teorías del cambio educativo con que contamos son más bien una cierta crónica de lo que hemos aprendido de los fracasos que una guía para conducir al éxito. Como dijo Sarason (2003, p. 29):

La escuela se ha resistido al cambio y a alcanzar los objetivos establecidos por los reformadores. Uno de los principales fracasos ha sido la incapacidad de los reformadores para enfrentarse a esta resistencia. Como resultado, cada nueva ola de reforma no aprende nada de los esfuerzos anteriores y hace 
recomendaciones que ya han fracasado en el pasado. Lo que se denomina reforma se basa en una aceptación del sistema tal cual ha sido y es. El cambio no puede suceder a menos que haya una alteración de las relaciones de poder entre aquellos que se encuentran en el sistema y dentro del aula.

La complejidad del cambio educativo le lleva a uno de los más importantes teóricos a afirmar que "jamás habrá una teoría definitiva del cambio. Es imposible teórica y empíricamente generar una teoría que sirva para todas las situaciones. No se conocen teorías definitivas del cambio, porque no existen ni pueden existir" (Fullan, 2004, p. 36). Al respecto, sería oportuno distinguir, como hace Escudero (2004), entre "teorías del cambio", capaces de analizarlo y comprenderlo, y "teorías para el cambio", capaces de trazar los trayectos por los que haya de discurrir para la mejora de la educación. Si bien contamos con un amplio corpus de la primeras, no hay caminos expeditivos que, empleando determinadas estrategias, conduzcan inexorablemente a la mejora.

En un contexto de incertidumbre, de falta de estabilidad y entornos turbulentos, cuando la planificación moderna del cambio y su posterior gestión han perdido credibilidad, se confía en movilizar la capacidad interna de cambio (de las escuelas como organizaciones, de los individuos y grupos) para regenerar internamente la mejora de la educación. En esta coyuntura postmoderna, se aduce, que las organizaciones con futuro serán aquellas que tengan capacidad para aprender (Bolívar, 2000a). De este modo, se pretende - en lugar de estrategias burocráticas, verticales o racionales del cambio - favorecer la emergencia de dinámicas autónomas de cambio, que puedan devolver el protagonismo a los agentes y, por ello mismo, pudieran tener un mayor grado de permanencia.

\section{La política educativa y su impacto en la enseñanza}

Habría que desarrollar políticas que mantengan el frágil equilibrio entre los estándares externos que estimulen la mejora y la autonomía escolar que constituya el motor del cambio interno. Desde mi punto de vista, una de las mejores vías para lograrlo consiste en determinar estándares profesionales en relación con el aprendizaje, la enseñanza y la organización escolar, y utilizarlos para orientar procesos de investigación en los centros que estimulen 
la participación, el aprendizaje y la renovación en su seno. (DarlingHammond, 2001, p. 289)

Si tenemos constatado que la mejora no suele ocurrir como consecuencia de un mandato político ("policy cannot madate what matters" se ha convertido ya en un cierto lema), tampoco podemos confiar en que florezca por generación espontánea de los propios centros, sin estímulos y apoyos de la administración educativa y comunidad. La cuestión, más bien, tal como la vemos hoy, es cómo las fuerzas exógenas puedan activar y sostener las dinámicas endógenas de la escuela: "no es plausible una visión dura de la reforma emprendida desde arriba, ni tampoco otra romántica dejada al albur de los cambios espontáneos desde las bases. Son necesarias tanto la imaginación local como el liderazgo político", asevera Darling-Hammond (op. cit., p. 274). Si la política educativa es sólo un instrumento de cambio, y no necesariamente el más poderoso, también hay el peligro de subestimar lo que las iniciativas políticas pueden aportar para mejorar la práctica docente.

Una cosa es el papel de la política educativa en países donde está plenamente demandado y justificado que lo primero es proporcionar mayor escolarización en unas condiciones más dignas, y otra cuando conseguido plenamente lo primero - de lo que se trata es de promover la mejora. La política de la administración educativa ha tenido una debilidad por concentrarse en reformas estructurales, que, por sí mismas, no cambian los modos de hacer ni contribuyen a potenciar la capacidad organizativa de desarrollo de los establecimientos de enseñanza. Al centrarse en grandes variables o factores remotos, suele olvidar o minusvalorar justamente los más relevantes: el apoyo a la puesta en práctica y, más ampliamente, potenciar el desarrollo organizacional de la escuela y el personal y profesional de los docentes. Desde los estudios sobre la implementación de reformas en los setenta sabemos que el cambio educativo es más fruto de factores locales que de la política central. Como consecuencia del conocido gap entre las políticas gubernamentales y la mejora de la escuela, se puede afirmar que son menos efectivas de lo que se creía y que muchos esfuerzos de mejora tienen que nadar contra las habituales regulaciones gubernamentales (Hopkins y Lewin, 2000).

Hay, pues, una especial tensión entre medidas a nivel central y en qué grado puedan potenciar o frenar el trabajo de los profesores. 
¿Donde situar los esfuerzos de mejora?: política educativa, escuela y aula

Una cierta ironía o paradoja lleva a que algunas iniciativas políticas intensificadoras inhiben los esfuerzos de mejora, perdiendo el potencial de sinergia que debían tener sobre centros y profesorado para, en último extremo, mejorar los niveles de aprendizaje del alumnado. Dado que lo relevante en educación no puede ser prescrito, porque, al final, será filtrado o modulado por centros y personas, la "nueva" política debe tender a "desarrollar las capacidades necesarias para llevar a cabo el trabajo requerido, así como también un compromiso firme y sostenido con el mismo, en lugar de presumir que sus directrices, sin más, vayan a provocar las nuevas ideas y prácticas planteadas", señala con razón DarlingHammond (op. cit., p. 278).

Si las políticas lineales de imposición burocrática están ya definitivamente desacreditadas por la práctica e investigación educativas, como decíamos, esto no significa que la política educativa no tenga un alto papel que jugar. En un contexto de retraimiento del papel de la Administración educativa para, en función de descentralización y autonomía (unido a la ideología neoliberal en auge), cederlo a los clientes o a los propios centros, no es menos politica lo que se precisa, sino más y mejor política. Aprendiendo del conocimiento acumulado de los fracasos, es preciso reinventar la política habitual en una "nueva" política, como dice en un clarificador planteamiento Darling-Hammond (2001), informada por el conocimiento de cómo las escuelas mejoran y, a la vez, capaz de movilizar las energías de los centros y coordinar los distintos componentes del sistema. Como reclama la misma autora, hace falta un

(...) nuevo paradigma de enfocar la política educativa. Supondría cambiar los afanes de los políticos y administradores, obsesionados en diseñar controles, por otros que se centren en desarrollar las capacidades de las escuelas y los profesores para que sean responsables del aprendizaje y tomen en cuenta las necesidades de los estudiantes y las preocupaciones de la comunidad. (Darling-Hammond, 2001, p. 44)

En efecto, la creciente tendencia a la descentralización y demanda de autonomía podría llevarnos a pensar que el papel de la política gubernamental es pasivo o, a lo sumo, supervisor mediante la evaluación en el control de resultados. En lugar de la amenaza de una regulación mercantil por los clientes (mediante los "cheques escolares" o cualquier otro dispositivo), defendemos, por contrario, un papel de la acción pública como actor de primer orden, eso sí resituada en un escenario distinto. 
Hoy sabemos que no bastan acciones políticas parciales, fragmentadas y cambiantes, ni tampoco grandes reformas que trastoquen el sistema cada cinco o diez años, son precisas acciones políticas comprehensivas y coherentes. El asunto es determinar qué intervenciones pueden mejorar más efectivamente la calidad de la enseñanza y diseñar la futura política en una forma globalizada y consistente en el tiempo.

Situados entre una lógica de acción burocrática y una lógica profesional, la gestión por proyectos pretende reforzar dicho papel de la escuela educativo como unidad estratégica de la mejora. En cualquier caso, en países con fuerte tradición centralista, ya lo hemos apuntado, el centro es un proyecto a construir, más que una realidad dada (Costa, 2003). Así, en estos países, como en el contexto español, nos hemos encontrado con una doble paradoja. Por dicha tradición histórica acumulada, nuestros establecimientos educativos están fuertemente uniformados, por lo que otorgarles protagonismo en el pilotaje de la mejora supone oponerse a dicha tradición burocrática y uniformadora. Por otra, en el grave estado de desvertebración a que han llegado últimamente, delegar los esfuerzos de mejora a las propias escuelas, por un lado, no conduce muy lejos, al imponerse la fuerza de la inercia de un sistema burocrático y formalista. Finalmente, podría significar ceder el asunto a una lógica de regulación mercantil, en un modo de gestión contaminado por lo privado.

La política educativa, para tener una incidencia en la mejora, debe, en primer lugar, ser coherente, "sistémica", con un foco claro y sostenido en el tiempo. Como ha resaltado Fullan (2000a), el problema no son las reformas, sino más bien la presencia de muchas iniciativas ad hoc, con políticas descoordinadas e innovaciones fragmentadas. Además, todos los componentes (desarrollo profesional, cambios curriculares, recursos etc.) deberán estar alineados de modo concertado en torno a dicho foco, y esto hacerlo en un período largo de tiempo (Cohen y Hill, 2001). Así, una iniciativa de reforma sistémica dará lugar a unos estándares curriculares perseguidos que promuevan unos niveles de aprendizaje de los estudiantes. En torno a ello se pondrán para apoyarlos los demás componentes.

Los cambios organizativos a nivel de escuela (estructuras de colaboración y procedimientos de toma de decisiones) deben ser aquellos que hacen posible la mejora a nivel de aula, apoyando y estimulando el trabajo del profesor en clase, y no en variables distantes o remotas, como frecuentemente ha ocurrido. A su vez, una escuela efectiva precisa de apoyos y presiones de la administración educativa de forma que, 
sin ahogar su propia dinámica de desarrollo, pueda potenciarla. Si el reto es difícil no estamos ciegos sobre cómo lograrlo, contamos con un amplio conocimiento acumulado, especialmente sobre qué no debemos hacer. Si la política educativa no debe renunciar al papel de liderazgo que le corresponde deberá hacerlo de modo que no ahogue las iniciativas de las escuelas.

Es cierto que, mientras contamos con evidencias empíricas fuertes de cómo un profesorado bien cualificado marca diferencias en el aprendizaje de los estudiantes, no tenemos documentado cómo las políticas educativas a gran escala contribuyen a capacitar a los profesores $y$, al tiempo, los niveles de consecución de los alumnos. No obstante, contamos con bases de conocimiento suficientes para sugerir vías de acción. Por un lado, es reconocida de modo general la necesidad de rediseñar la profesión docente y el trabajo en los centros en formas y modos para que sean posibles las prácticas educativas que debamos pretender. No basta crear un entorno positivo de trabajo, es preciso contar con buenas visiones de a dónde queremos ir, concretadas en estándares deseables a conseguir que, sin caer en estandarización (ese es el grave peligro a evitar) y debidamente consensuados, puedan proveer direcciones, incentivos y exigencias de lo que los profesores deben enseñar y los alumnos aprender. Una rémora del pensamiento progresista en educación ha sido oponerse a cualquier forma de control sobre centros y profesores. Pero es difícil imaginar cómo garantizar el derecho de aprender de todos si no hay arbitrados dispositivos para garantizar que las escuelas den cuentas de la educación ofrecida. Otro asunto es cómo hacerlo, para que no quede en una estrategia de "palos y zanahorias" ni caigan en una estandarización para la elección de los clientes, sino en formas que potencien la mejora con los recursos oportunos (Bolívar, 2003).

\section{2. ¿Estándares externos para promover la mejora interna?}

Dentro de la presión por la mejora, entendida como incremento de los niveles de aprendizaje de los alumnos, el movimiento de reforma basado en estándares deseables (standards-based reform) está alcanzando el carácter de una nueva "ola" en toda la literatura y práctica anglosajonas o, como dice Hargreaves (2003), configurándose como una "nueva ortodoxia del cambio educativo". En Norteamérica lleva ya una década, y en Inglaterra ha adquirido nuevos bríos con la dirección 
por David Hopkins de la Unidad de "Estándares y Efectividad". Como sumariza Goertz (2000, p. 65),

El propósito de la estrategia de reforma basada en estándares es proveer apoyo desde el top-down (nivel estatal) a la reforma bottom-up (nivel del centro). La estrategia tiene tres componentes principales: una visión y objetivos unificados que provean una coherente dirección sistémica a la educación. El Estado debe establecer metas de resultados de los estudiantes que enfoquen primariamente la enseñanza y aprendizaje que debe acompañar los altos estándares. En segundo lugar, la estrategia es una guia de sistema coherente de política educativa que promueva estos resultados ambiciosos de los estudiantes. Esto implica la coordinación de aspectos claves de la política educativa que afectan a la enseñanza y aprendizaje: currículum, materiales, formación del profesorado, y evaluación (...). La tercera línea de acción es un sistema de gobierno reestructurado que defina las responsabilidades de los diversos niveles del sistema para facilitar la adopción a nivel de aula de nuevos contenidos y metodología.

Si una clave del funcionamiento de la escuela, como pusieron de manifiesto los análisis institucionales, es que su estructura está "débilmente acoplada", funcionando cada uno independientemente en el espacio privado de su clase, la reforma basada en estándares ataca este punto en su base: los profesores deben esforzarse en el aula para conseguir las metas fijadas a nivel estatal en los alumnos, y dar cuenta de ello a nivel de escuela. Por ello, declara Elmore (2000, p. 4): "se quiera o no, la reforma basada en estándares representa un cambio fundamental en la relación entre política y práctica docente". Cada escuela tiene autonomía para desarrollar el currículum, pero - mediante el rendimiento de cuentas (accountability) - deberá preocuparse por conseguir, de modo conjunto con sus colegas, los estándares establecidos. Es el nuevo modo (re)centralizador de presionar políticamente, al determinar lo que los estudiantes deben aprender y dominar.

Hay supuestos en el movimiento del rendimiento de cuentas demasiado simples, cuando no ingenuos: como consecuencia de los resultados (y publicidad) de las evaluaciones, los diferentes actores concernidos (por las sanciones o incentivos consiguientes) necesariamente se esforzarán por mejorar. Pero hay pocas evidencias de que el rendimiento de cuentas de centros y profesores mediante tests provoque, por sí mismo, una mejora de los resultados educativos. Aquellos centros que se encuentran fracasados difícilmente van a encontrar un incentivo en ver reflejada su situación en los últimos lugares y, en los restantes, si no hay 
¿Donde situar los esfuerzos de mejora?: política educativa, escuela y aula

creados procesos previos de análisis y revisión, escasa incidencia van a tener las evaluaciones externas para su mejora. Como muestra Hargreaves (2003, p. 100), al describir casos de escuelas (Nueva York, Ontario) sometidas a la reforma basada en estándares, en la vida de los centros éstos han degenerado en una estandarización, que premia a los mejores y degrada o culpabiliza a los centros situados en zonas marginales: "es irrelevante para las escuelas que obtienen mejores resultados, a la vez que aumenta la exclusión de aquellos centros y estudiantes que obtienen peores resultados, que encuentran los estándares desesperadamente fuera de su alcance".

Sin embargo, además de una posición crítica por la forma y usos que suele conllevar, cabe defender - más progresistamente - que el sistema educativo no puede garantizar el derecho de aprender para todos o, lo que es lo mismo, una "educación democrática", si no se fijan unas metas o estándares a alcanzar, y se evalúa su grado de consecución en las escuelas. De hecho, puede ser un modo para que las cuestiones de la práctica y su mejora no queden recluidas al privatismo del aula, pues el rendimiento de cuentas por niveles de consecución requiere el desarrollo de una práctica de mejora escolar continua, un cuerpo de conocimientos acerca de cómo incrementar la calidad de la práctica docente y estimular el aprendizaje de los alumnos a gran escala en las diferentes aulas, escuelas, y el sistema escolar en su conjunto. El asunto, como siempre, dependerá de cómo se lleve a cabo dicho control y los recursos dispuestos para la mejora. Por eso, un factor crítico del éxito es la adecuada combinación de serias exigencias externas con dispositivos que desarrollan la capacidad interna.

El tema es cómo establecer estándares (y la correspondiente evaluación) que definan niveles de consecución deseables, según cada contexto (sin estandarización), y una evaluación periódica que asegure una educación de calidad para todos y, al tiempo, proporcione los medios considerados necesarios para conseguirlo. Y es que, como argumenta Darling-Hammond (2001, p. 314),

si se aspira a que los alumnos alcancen unos estándares de mayor calidad educativa hay que suponer que también los profesores han de satisfacer ciertos estándares o criterios de calidad en su trabajo. Unos estándares de enseñanza elevados y rigurosos constituyen la piedra angular de un sistema de control que concentre su atención en el aprendizaje de los alumnos. 
Hay, no obstante, razonables dudas sobre si la evaluación externa de los resultados (evaluación como producto) pueda comportar un proceso de mejora interna (Adams y Kirst, 1999). Una política evaluadora de premios y castigos lleva poco lejos. Por su parte, Elmore (2002) ha establecido el "principio de reciprocidad" en el rendimiento de cuentas, consistente en que las exigencias de consecución de estándares tienen, recíprocamente (quid pro quo), que corresponderse con la capacitación para lograrlos. Si se establece un control de niveles de consecución exige, recíprocamente, poner los medios, recursos e incentivos que hagan posible la mejora. Por eso, la presión actual por la "accountability", situada en sus justos términos, como hace Elmore (2002, p. 5), exige que el sistema educativo proporcione la capacidad necesaria para responde a dichas demandas:

A fin de que la gente en las escuelas pueda responder a las presiones externas de accountability, tienen que aprender a hacer su trabajo de modo diferente y a reconstruir la organización de las escuelas sobre otros modos diferentes de hacer el trabajo. Si el público y los políticos quieren incrementar la atención sobre la calidad académica y resultados, el quid pro quo es invertir en el conocimiento y las destrezas necesarias para producirlo. Si los educadores quieren legitimidad, propósitos y credibilidad para su trabajo, el quid pro quo es aprender a hacer de modo diferente su trabajo y aceptar un nuevo modelo de accountability

La reforma basada en estándares no puede olvidar una pieza clave: la capacidad para conseguirlos, si es que se quiere apostar por ellos. Las presiones y apoyos de la política educativa desde arriba y las energías de abajo se necesitan mutuamente. Si es preciso crear visiones de las metas a alcanzar, y estándares que definan el progreso en su consecución, recíprocamente la política educativa se debe poner al servicio de asegurar los conocimientos y habilidades del profesorado para que los alumnos alcancen altos niveles de comprensión en el aprendizaje. Si no se hace (como suele suceder), evidentemente aboca a una estandarización e incremento de las desigualdades, al faltar los recursos para llevarlos a cabo.

Para concluir, como analiza Eisner (2002, p. 252), con la sabiduría que le proporciona la larga experiencia vivida, podemos decir que es "un esfuerzo bienintencionado pero conceptualmente superficial para mejorar las escuelas. Mi propósito es poner en evidencia que la iniciativa en la que estamos embarcado no es novedosa; ya la emprendimos antes", 
con el tema de determinar los objetivos. Esto le hace pensar, aparte de las dificultades en su determinación y aplicación, si no nos desvía de los problemas fundamentales de las escuelas, distrayendo la atención y que, al final, quede como otra de las tantas olas que pasan.

\section{La escuela como unidad estratégica del cambio. Una idea a revisar y resituar}

En los ochenta, por un conjunto de factores y desde diferentes ángulos confluyentes (Bolívar, 1999), se pasó a entender que es la labor conjunta de la escuela la clave de la mejora y/o "eficacia", convirtiéndose - desengañados de que el cambio pueda venir desde arriba - en un lugar estratégico del cambio educativo. Si bien no se olvidaba que la mejora de los aprendizajes de los alumnos es la misión última que justifica la experiencia escolar, se hace depender de la labor conjunta de toda la escuela. Ahora prioritariamente, los procesos de mejora deben iniciarse en dimensiones de segundo orden (a nivel de organización), para que los que sucedan, por implicación, a nivel de aula se sostengan de modo estable. Importa generar condiciones internas en los establecimientos escolares que promuevan su propio desarrollo como organizaciones. La asistencia y asesoramiento debe, consecuentemente, dirigirse a generar la adquisición de la "metacapacidad" (competencia de segundo orden) de resolver por sí mismo los problemas organizativos o didácticos, conocida como capacidad interna de cambio.

Desengañados en parte de las soluciones a nivel "macro" (estructurales o políticos) y de las "micro" (tareas del aula), emergió con fuerza un paradigma meso, que sitúa - o, mejor, transfiere - a la propia escuela la solución de problemas. Cuando la articulación vertical de las decisiones fallan, o los programas de cambio no lo producen, sólo queda el recurso a la integración y coherencia horizontal. Así, hemos mantenido que el establecimiento escolar se puede, entonces, convertir en una "organización estratégica” entre las propuestas externas de reforma y la realización de la innovación en la práctica. La cultura organizativa de la escuela desempeña un papel mediador en la construcción del cambio, siendo más relevante que las fuerzas que vienen del exterior (Gather Thurler, 2001). Para esto, al menos, las escuelas deben tener la capacidad de decidir sobre los proyectos de cambio de acuerdo con sus propias prioridades, tienen que existir oportunidades de trabajar juntos y oportunidades 
de desarrollo profesional así como los recursos adecuados; y los procesos de mejora deben conectarse con las demandas y expectativas del entorno social inmediato.

Además de factores externos, algunas razones internas están dando lugar a resituar el lugar del centro y a reconceptualizar lo que decíamos de unidad de cambio. Así, la defensa decidida que en otros trabajos (Escudero y Bolívar, 1994; Bolívar, 1996) hemos hecho de un Desarrollo Curricular Basado en la Escuela, ahora nos parece una propuesta un tanto ingenua, apropiada para grupos de renovación pedagógica comprometidos por mejorar desde la base la educación que tenían a su cargo. Actualmente, por un lado, las presiones externas para conseguir determinados niveles se han acentuado, no sólo por las políticas educativas, sino por las demandas de las propias familias, crecientemente más clientes que ciudadanos. Antes, lemas como tomar propiedad del proyecto, colegialidad o desarrollo interno de la escuela, era un asunto interno que podría ser llevado a cabo, desentendiéndose de lo que demanda el entorno. Pero, a comienzos del nuevo milenio, ser insensible a dichas demandas puede significar la selección natural o progresiva muerte de la escuela.

Por otro lado, estimo, unos presupuestos de la ética de la modernidad (kantiana) bienintencionadamente nos hacían creer que lo determinado por el centro, si se hace de modo consensuado y de modo autónomo por los propios implicados, es de por sí bueno para toda la comunidad. Cuando no somos del todo modernos, desconfiamos de que la autonomía conduzca a la universalidad. Primero, porque tal autonomía es, en parte, irreal. De hecho, como se ha sugerido por distintos análisis, en muchos casos ha funcionado como una forma sutil (de "colonizar") para tomar como propios lo que no eran sino mandatos externos camuflados (es decir, en la práctica no existía verdadera autonomía). En otros, el profesorado - que son, como humanos y no ángeles, seres micropolíticos - pueden decidir en función de fines heterónomos.

La autonomía y descentralización, como incremento de la capacidad de toma de decisiones a nivel de centro, parece ser una condición estructural necesaria, aunque no suficiente, para implicar a los agentes en la toma de decisiones, en el compromiso colectivo y en el aprendizaje de la organización. Es una expectativa poco realista creer que la descentralización provoque por sí misma una mejora institucional de la acción educativa. Calhoum y Joyce (1998, p. 1293) calculan que "sólo sobre un diez por ciento de las escuelas han tenido capacidad para generar ini- 
¿Donde situar los esfuerzos de mejora?: política educativa, escuela y aula

ciativas que hayan cambiado sustantivamente las dimensiones curricular, instructiva y tecnológica de la escuela”. Se han aducido un conjunto de hipótesis para explicar por qué el enfoque basado en la escuela no ha hecho cambios significativos a nivel curricular e instructivo. Así, en algunos casos, se ha tomado como un fin en sí mismo, y no un medio para mejorar la educación de los alumnos, o pocas escuelas han hecho de la colegialidad algo que modifique sustantivamente la estructura normativa de la escuela.

Transferir la mejora a las iniciativas de la propia escuela no es, en cualquier caso, una panacea. Conduce poco lejos decretar una autonomía para dejar que se las arreglen por sí solos, sin generar capacidades. En estos casos, suele quedar como una retórica con fines legitimatorios, como decía Weiler (1996). Al contrario, potenciar la coordinación horizontal no supone que la coordinación vertical no tenga un papel clave en estimular las dinámicas endógenas de cada centro, ya sea mediante proyectos-contrato de autonomía, ya, sobre todo, por su apoyo decidido para que cada centro construya su propia capacidad de desarrollo y mejora. En consecuencia, prácticamente, cabe entenderla como la creación de dispositivos, competencias, apoyos y medios que permitan que las escuelas, en conjunción con su entorno local, puedan construir su propio espacio de desarrollo, en función de unos objetivos asumidos colegiadamente y un proyecto, si es posible, contratado con la administración o comunidad.

Así, en los procesos de mejora escolar, se ha hablado, un tanto imprecisamente, de que lo que importa es mejorar la escuela "como totalidad”, cuando es difícil definir y articular qué significa esto (Bolívar, 2001). Así, al analizar la realidad de vida organizativa de los Institutos de Secundaria (González, 2004) se ha mostrado que el establecimiento escolar como unidad en colaboración no existe propiamente, cuando los departamentos son las unidades básicas de organización y de la identidad profesional. La división por especialidades y Departamentos no sólo constituye el referente básico de la identidad profesional e interacción social, sino que, como comentan Talbert, Mclaughlin \& Rowan (1993, p. 55), "la colegialidad de los profesores, la extensión de la colaboración y el apoyo entre el profesorado varían considerablemente según Departamentos en el mismo Instituto". Por tanto, no se puede hablar de la escuela como unidad, pues aparte el excesivo número de profesores en estos centros escolares - es en el interior de los Departamentos donde cabe, en primer lugar y de modo diferencial, la acción conjunta y la colaboración. 
Por su parte, si el desarrollo de una cultura de colaboración se convirtió - desde fines de los ochenta - en mensaje salvífico, cual discurso redentor y nueva ortodoxia, para mejorar la educación, reapropiada dentro de la retórica del discurso de la política educativa, las relaciones en colaboración se han visto como un potente dispositivo para aprender y resolver problemas, construir una solidaridad y cooperación en el centro escolar y satisfacer la necesidad de apoyo mutuo de los profesores. Además de una vía de desarrollo profesional, por lo que aquí nos importa, podía ser una variable mediadora para mejorar la educación de los alumnos, pues cuando los profesores intercambian experiencias y conocimiento crecen profesionalmente. Como he analizado en otro lugar (Bolívar, 2000b), también este campo se está viendo sometido a una fuerte reconceptualización, una vez que se abrió, la "caja negra” de la colaboración (Fullan, 2004). Por un lado, la individualidad tiene que seguir manteniendo toda su relevancia, pues un centro funciona bien cuando tiene buenos profesionales, aunque tengan pocas oportunidades de trabajo común. Por otro, la participación en la toma de decisiones, en muchos casos, se ha constatado no se centra en lo fundamental: el currículum, cómo es enseñado, y cómo incrementar la educación de los alumnos.

En su lugar, hemos aprendido con el tiempo que cualquier cambio debe afectar al "núcleo duro" de la enseñanza, entendido como los modos en que los profesores comprenden la naturaleza del conocimiento y se intercambian dichos conocimientos con los colegas, el papel que tienen los alumnos en los procesos de enseñanza, así como estas ideas sobre conocimiento y enseñanza se manifiestan en el aprendizaje en el aula. Para afectar (y alterar) ese "núcleo", sin duda debe incluir cambios organizativos, es decir una estructura favorable para la mejora escolar, tales como reordenación de las escuelas, distribución de las clases, agrupamientos de alumnos, responsabilidades de los profesores, relaciones entre los profesores en su trabajo cotidiano, así como el proceso de evaluar el aprendizaje y comunicarlo a otras partes. En caso contrario, serán asimilados/acomodados a los modos anteriores de hacer (estructura superficial).

\section{El núcleo de la mejora: procesos de enseñanza-aprendizaje en el aula}

Si como venimos argumentando desengañados de que la mejora pueda venir desde arriba por buenos diseños para, en su lugar, confiar en la labor conjunta de la escuela como clave de la mejora y lugar estratégi- 
co del cambio educativo, el trabajo en el aula quedó en un segundo plano dependiente del conjunto. A mediados de los noventa, con la presión por los resultados de aprendizaje, en una cierta vuelta, el aula y los procesos de enseñanza y aprendizaje se erigen en el núcleo de cualquier propuesta de cambio, pues lo que realmente importa es asegurar el aprendizaje de todos los alumnos.

Si ya no se puede, definitivamente, confiar en iniciativas centrales, tampoco las "bottom-up" (de abajo-arriba), por sí mismas, son la vía de salvación. Es mejor, pensamos ahora, dentro de lo que ha dado en llamarse tercera "ola" o fase, centrarse en aquellas variables próximas al aula, que son las que pueden conducir a la implementación efectiva, pues, en último extremo, es lo que los profesores hacen en clase lo que marca la diferencia en los resultados de aprendizaje de los alumnos (Hopkins y Reynolds, 2001). En fin, en un cierto viaje de ida y vuelta estamos volviendo a lo que siempre es el núcleo de la acción docente: los procesos de enseñanza-aprendizaje en el aula. Si una innovación (curricular, organizativa o profesional) no incide en la calidad de aprendizaje de los alumnos, difícilmente podríamos calificarla de mejora. Por eso, el foco de prioridad debe ser incrementar la calidad de educación recibida por todos los alumnos en condiciones de equidad en todas las escuelas.

De este modo, visto que algunos de los esfuerzos emprendidos a nivel de escuela (tanto dentro los procesos de mejora de la escuela como de reestructuración escolar) no han incidido significativamente en el aprendizaje de los alumnos, se ha resaltado el aula o clase como un nivel central, al que deben subordinarse los esfuerzos de mejora emprendidos; aún cuando precise el apoyo congruente de los restantes (Ainscow et al., 2001). Estamos, pues, revaluando si no ha existido una cierta ingenuidad y pobre conceptualización de los modos precisos en que rediseñar la estructura organizativa de los centros o la colaboración entre el profesorado podía impactar en el nivel de la enseñanza en el aula. El discurso sobre estrategias metodológicas (modelos de enseñanza, prácticas efectivas de enseñanza etc.) ha estado un tanto silenciado y algunas direcciones de reforma han dejado en un segundo plano la práctica docente en el aula.

Aparte de los cambios de acento, propios de cada momento, el grupo de Hopkins, (auto)revisando la mayoría de sus experiencias de mejora de la escuela, apuntan que el nivel del aula estaba pobremente 
conceptualizado como para que pudieran tener un impacto directo en el incremento del aprendizaje del alumnado:

De acuerdo con nuestra experiencia, una mayoría de iniciativas de mejora escolar han sido pobremente conceptualizadas en aquellos modos precisos en que podrían tener impacto en el aprendizaje en el aula, que es el factor educativo que mayor impacto pueda tener en resultados de los alumnos. Mientras muchas escuelas conjugan los niveles de currículum y organización, los modos precisos en que estos cambios van a impactar en el aprendizaje son poco claros y normalmente mal dirigidos. (Hopkins y Levin, 2000, p. 21)

Haciendo un balance de la travesía recorrida, pues, de adquirir en los ochenta toda su relevancia la escuela como organización, revisiones en los noventa han hecho caer en la cuenta de que no es posible desdeñar los aspectos didácticos y metodológicos del aula, que es donde en último extremo se juega la mejora. A este respecto, la tradición de "escuelas eficaces", una vez reformulada en los noventa de modo más comprehensivo, ha contribuido decisivamente a focalizar las estrategias de apoyo y mejora en los aspectos de enseñanza-aprendizaje. Dentro de esta tradición contamos ya con un amplio cuerpo de evidencias sobre los "efectos" de la escuela en el aprendizaje de los alumnos, así como se han hecho buenas contribuciones sobre formas metodológicas (cuantitativas y cualitativas) de medirlos y/o apreciarlos. Los cambios a nivel de centro deben ser complementarios a cambios a nivel de prácticas docentes, el aprendizaje individual con el aprendizaje a nivel de organización. De este modo, como ya se ha señalado, el referido grupo que, en otro tiempo, centraba la mejora a nivel de centro, estipulan ahora:

Adoptamos un enfoque del cambio educativo que se centra en el rendimiento del alumno $y$ en la capacidad de la escuela para afrontar el cambio. Denominamos a este enfoque concreto mejora de la escuela; consideramos la mejora como un enfoque definido del cambio educativo que destaca los resultados del alumno asi como la capacidad de la escuela para dirigir iniciativas de mejora. En este sentido, la mejora de la escuela se refiere a la elevación del rendimiento del alumno, centrándose en el proceso de enseñanza-aprendizaje y en las condiciones que lo respaldan. (Ainscow et al., 2001, p. 11)

En el ámbito anglosajón, que, por otra parte, ha marcado al menos la investigación educativa en los restantes países, se han distinguido tres "olas" de mejora: dejando la primera de política centralizada, de una 
"segunda ola" (reestructuración) que se dirige a rediseñar la estructura de la organización de los centros y el ejercicio de la profesión docente, a una cierta tercera ola, donde se da un paso más en el rediseño organizativo, para poner el foco en el aprendizaje de los alumnos, sin el cual no cabe calidad de la enseñanza para todos (Tabla 1).

\section{Tabla 1}

(Diferencias entre dos últimas "olas" en la mejora)

\begin{tabular}{|l|l|l|}
\hline & $\begin{array}{l}\text { "Segunda ola" } \\
\text { (1986-95) } \\
\text { ESCUELA }\end{array}$ & $\begin{array}{l}\text { "Tercera ola" } \\
\text { (1996 y ss.) } \\
\text { AULA: BUENAS ESCUELAS }\end{array}$ \\
\hline Mejora & $\begin{array}{l}\text { Descentralizada: protagonismo a las } \\
\text { escuelas y profesorado. } \\
\text { Compromiso de los agentes. }\end{array}$ & $\begin{array}{l}\text { Aula: rediseñar con el foco en un aprendizaje } \\
\text { de calidad de todos los alumnos. }\end{array}$ \\
\hline Política & $\begin{array}{l}\text { Autonomía y gestión basada en la escuela. } \\
\text { Reestructurar los centros escolares. }\end{array}$ & $\begin{array}{l}\text { Nueva política activa que estimule y capacite } \\
\text { a centros y profesorado. }\end{array}$ \\
\hline Profesorado & $\begin{array}{l}\text { Reprofesionalización y capacitación. } \\
\text { Agentes activos. }\end{array}$ & $\begin{array}{l}\text { Recrear la profesión: Su formación y } \\
\text { competencia factor crítico de la mejora. }\end{array}$ \\
\hline Currículum & $\begin{array}{l}\text { Reconstrucción por las escuelas. } \\
\text { Enseñanza para la comprensión. }\end{array}$ & Definir estándares, sin estandarización. \\
\hline
\end{tabular}

A su vez, las evaluaciones de la gestión basada en el centro, a mediados de los noventa, muestran que ésta ha tenido una débil relación con la mejora de la práctica docente. Las conclusiones obvias sugieren focalizarse, en su lugar, en los cambios a nivel de aula, y tomar los cambios organizativos como un "instrumento" a su servicio para acompañarlos. Cualquier esfuerzo reestructurador de los centros educativos ha de estar centrado en cómo afecta para mejorar el aprendizaje de los alumnos y qué condiciones requiere para que ocurra. Es algo razonable pensar que "seguramente los cambios estructurales en las escuelas se han realizado con la intención de producir cambios en la enseñanza y el aprendizaje" (Elmore, 1995b, p. 23). La reestructuración de la escuela se tiene que dirigir a la mejora del aula. Pero es algo que no se puede dar por supuesto. Y la investigación al respecto (Elmore, Peterson y McCarthey, 1996; Leithwood y Menzies, 1998) es más bien pesimista al respecto o, al menos, mantiene que el asunto es más complejo. El éxito de esta "segunda ola” de reformas, en último extremo, sólo puede medirse si tiene una in- 
cidencia en cómo los profesores piensan e interactúan con los alumnos en el aula. Cambiar los modos en que están organizados las escuelas no se justifica si no da lugar a una transformación de la enseñanza y aprendizaje. Pero, es evidente que, siendo una condición necesaria, no es suficiente, pues no es primariamente un asunto organizativo, sino cultural: nuevos modos de pensar y hacer, en unos contextos provocadores del aprendizaje conjunto del profesorado.

Por su parte, decretar la autonomía ha llevado poco lejos, aparte de su uso al servicio de una ideología mercantil, si no se crean condiciones que permitan "construirla" por cada establecimiento educativo. La mejora se juega no en la estructura, sino en la cultura escolar (hábitos, habilidades, formas de hacer), aun cuando determinadas estructuras puedan bloquear o facilitar el proceso. Por un lado, la mayoría de los cambios educativos se han dirigido a las estructuras o al currículum pretendido, pero no han llegado a afectar al núcleo duro de la práctica educativa; por otra, crecientemente se ha reconocido la complejidad del cambio en educación, mostrando en qué grado los cambios curriculares planificados se puedan ver reflejadas en lo que ocurre en los centros y aulas. Si bien se requieren cambios organizativos y estructurales a gran escala, éstos por sí mismos no provocan la mejora. Sin alterar los modos de pensar y hacer ("reculturizar"), cultivando la implicación activa de los docentes, las reformas estructurales llevan poco lejos. Y justamente esto (cambiar las mentes o conseguir compromisos) es lo que las reformas por sí mismas no pueden hacer. "Transformar la cultura - cambiar el modo en que hacemos las cosas - es el punto esencial. Lo llamo reculturizar", señala Fullan (2002c, p. 58).

Mientras tanto, una buena y coherente política educativa debe contribuir a que cada centro construye su propia capacidad de desarrollo y mejora. Ésta no es decretar una autonomía para dejar que se las arreglen por sí solos. Al contrario, potenciar la coordinación horizontal no supone que la coordinación vertical no tenga un papel clave en estimular las dinámicas endógenas de cada escuela. Además, esto supone, entre otros, reconocer que un profesorado capacitado es el factor crítico para una mejor educación del alumnado, lo que implica, entre otras, la preparación de buenos profesionales y formación continuada, desarrollar buenas visiones de la enseñanza y el aprendizaje, reorganizar los establecimientos escolares en modos que proporcionen una equidad en la diversidad. 
El foco (target) de mejora debe ser el incremento de los aprendizajes de todos los alumnos y, de acuerdo con él, demandar cambios organizativos o apoyos de la política educativa. Ahora bien, recogiendo lo que hemos aprendido en la travesía, la mejora a nivel del aula no puede mantenerse de modo continuo más que si está sostenido por un equipo y trabajo en colaboración a nivel de escuela. Parece claro que, sin haber generado una capacidad interna de cambio (asunto clave heredado de la "mejora de la escuela”), los esfuerzos innovadores de profesores están condenados a quedar marginalizados o a tornarse inefectivos. Expandir la visión del aula a la escuela como conjunto es un paso necesario, que suele ser facilitado por trabajo en equipo en torno a proyectos propios.

\section{5. ¿En qué quedamos, tras la travesía recorrida?}

Estamos, entonces, en condiciones de hacer un cierto balance provisional de lo que han dado de sí los esfuerzos de mejora emprendidos. Más allá de las modas discursivas que recorren el mundo educativo, es evidente que cualquier esfuerzo de mejora se debe dirigir al currículum y procesos de enseñanza en el aula (el instructional core, que ha dicho Elmore), que ahora creemos, con razón, debe verse arropado (y sostenido) a nivel de cada establecimiento educativo, y, más ampliamente, por medidas congruentes de la política educativa y apoyo de agentes de cambio externos e internos. Nada puede sustituir la necesidad de promover el desarrollo interno de las escuelas, pues sin su concurso la mejora no sucederá.

Es también una lección aprendida que focalizarse exclusivamente en la enseñanza y el aprendizaje no es una condición suficiente para la mejora. Como bien expresó Elmore (1995a, p. 366):

Los principios de la buena práctica docente tienen dificultades de arraigar en los escuelas por dos razones esencialmente: (a) requieren conocimiento del contenido y habilidades pedagógicas que presumiblemente pocos profesores poseen, y (b) cambiar ciertas pautas básicas en la organización de la escuela. Ningún problema puede ser resuelto independientemente del otro, ni la práctica docente es probable que cambie en ausencia de soluciones que operen simultáneamente en ambos frentes.

No hay soluciones simples ni mágicas, el proceso de cambio es siempre complejo y contingente, en parte caótico, dependiente de contextos, centros y política, como para ser captado con un único modelo; con- 
cluye Fullan (2004). De ahí la vulnerabilidad de soluciones empaquetadas. En una vuelta al sentido común, si las políticas educativas no pueden cambiar lo relevante en la mejora (cómo los profesores enseñan y los alumnos aprenden), sí puede crear condiciones para el aprendizaje y desarrollo de las escuelas. Se impone una reciprocidad en una calle de doble sentido.

La mejora del aprendizaje de los alumnos, en un contexto enriquecido y equitativo, supone, conjuntamente, un aprendizaje profesional y un aprendizaje del sistema (Knapp et al., 2003). Esta interacción interniveles, sin duda, se ve apoyada por un entorno que introduzca impulsos, en especial un liderazgo "distribuido" que promueva y estimule la mejora. Si el elemento central (target) es el aprendizaje de los estudiantes, se deben rediseñar aquellas estructuras que hacen posible la mejora a nivel de aula, apoyando y estimulando el trabajo del profesor en clase. A su vez, un centro efectivo precisa de apoyos y presiones de la administración educativa de forma que, sin ahogar su propia dinámica de desarrollo, pueda potenciarla. Ni la descentralización por sí misma soluciona los problemas ni, por supuesto, estrategias verticales funcionan.

El rendimiento de los alumnos depende, entonces, de la interacción de un conjunto de factores. Si la competencia del profesorado - y su incremento, mediante el aprendizaje profesional - es el fundamento de la mejora de la práctica docente, para que de modo continuado afecte al alumno a lo largo de los cursos escolares, debe integrarse en un trabajo colectivo de todo el centro. Este poder colectivo de todo el profesorado para mejorar el rendimiento de los alumnos puede ser reunido bajo el título de "capacidad de la escuela" (Newmann, King y Youngs, 2000). A su vez, el impulso último proviene del sistema (política educativa y programas) y la coherencia que mantenga.

Por un lado, parece claro que la política educativa debe apoyar la autonomía y descentralización, que no significa una delegación de responsabilidades, cuanto capacitarlos para tomar las decisiones que estimen más oportunas. Se trate de emplear

una dialéctica fuera-dentro que contribuya a estimular reformas desde dentro hacia fuera. Ambos sistemas, el conjunto de estándares relativos a la prestación del servicio educativo y los procesos de revisión de la calidad en los centros, son decisivos para garantizar que los alumnos dispongan de las oportunidades adecuadas para aprender según las exigencias de los nuevos estándares. (Darling-Hammond, 2001, p. 330) 
Siendo el foco de la política educativa incrementar la capacidad de desarrollo de los centros, esto no puede conducir a desentenderse de unas exigencias de calidad educativa para toda la ciudadanía. De ahí la exigencia (y evaluación) de estándares a lograr. Mientras tanto, se debe estimular la innovación, conectando debidamente dichos niveles (política, centro, aula).

Como conclusión, desde diferentes frentes (Fullan, 2002b; Darling-Hammond, 2001), se señala que se debe dar una combinación de perspectivas: de arriba-abajo (top-down), de abajo-arriba (bottom-up), pero también una recomposición horizontal o interna (inside-out). Parece claro, a nivel de teoría, que el blanco u objetivo central (the target) al que deben tender todas las acciones es - parece obvio en centros educativos - el aprendizaje de los alumnos. Éste se entiende, de acuerdo con Cohen y Ball (2000), como la interacción entre profesores, contenido y alumnos, dentro de un práctica docente determinada en un entorno. Esta interacción, pues, supone que lo que se aprende es dependiente de las relaciones particulares que se establecen entre profesores y alumnos (cómo se agrupan, dimensiones de los alumnos que se tienen en cuenta, papel del profesor en la interacción, cómo la enseñanza ha sido diseñada y planificada etc.), conjuntamente con el contenido. La práctica docente, entendida de modo amplio, se convierte, pues, en el blanco de la reforma política.

Si queremos llegar a tener mejores escuelas, es decir escuelas donde los estudiantes alcancen niveles más altos de aprendizaje, contamos con una cierta "teoría de la acción", que Fuhrman y Odden (2001), dos reconocidos especialistas, en una introducción a un monográfico sobre la reforma escolar, establecen en los siguientes principios directrices:

1. Debemos contar con metas claras y ambiciosas, juntamente con indicadores de resultados coherentes con estándares educativos y medidas de los logros de los estudiantes.

2. Cuando dichas metas ambiciosas pretenden incrementar los niveles de realización, la tecnología común de la educación (la práctica docente) debe cambiar drásticamente. Un fuerte foco en el cambio didáctico es necesario, cuando se quiere que los estudiantes consigan altos niveles de suficiencia. Para que esto suceda también se precisan cambios a nivel de organización.

3. Para conseguir una dramática mejora de la enseñanza en todas las escuelas requiere esfuerzos extensivos en formación permanente, en buenos materiales curriculares y en un liderazgo en el sistema y a nivel de escuela. El desarrollo 
de la capacidad no sucede espontáneamente, se requiere un fuerte liderazgo por la política educativa.

4. Semejante cambio sistémico requiere incentivos para proveer refuerzos positivos para que ocurra la mejora y para que las escuelas se esfuercen en más y mejor cambio. El rendimiento de cuentas debe ser parte de estas estrategias, no una reforma en sí misma.

En su excelente revisión, Louis, Toole y Hargreaves (1999) señalan la necesidad de reconfigurar la investigación y el conocimiento sobre la mejora escolar. Nuestro pensamiento sobre el cambio ha estado dominado por un conjunto de presupuestos que deben ser revisados o cambiados. Además de las demandas crecientes del entorno, debe existir presión y liderazgo político para movilizar "desde fuera" las energías internas. Hemos pecado de una cierta ingenuidad, en otros casos de "romanticismo", pensando que el contenido, objetivos y estrategias del cambio es asunto de los propios implicados. Si bien está demostrado que las estrategias centralizadas no funcionan (pues no se puede imponer lo importante del cambio), las descentralizadas, como la gestión local, por sí mismas tampoco. Nuestro dilema actual es combinar adecuadamente la presión externa a gran escala con la motivación interna a nivel local, por lo que "conseguir una reforma a gran escala basada en la autoría local es el nuevo reto del cambio educativo, dejando a un lado la falsa disyuntiva entre innovación local y reforma superficial" (Fullan, 2002b, p. 31).

Recebido em junho de 2005 e aprovado em agosto de 2005.

\section{Referencias bibliográficas}

ADAMS, J.E.; KIRST, M. W. New demands and concepts for educational accountability: striving for results in an era of excelence. In: Murphy. J.; Louis, S.K. (Ed.). Handbook of research on educational administration. 2. ed. San Francisco: Jossey-Bass, 1999. p. 463-489.

AINSCOW, M. et al. Crear condiciones para la mejora del trabajo en el aula: manual para la formación del profesorado. Madrid: Narcea, 2001.

BOLIVAR, A. El lugar del centro escolar en la política curricular actual: mas allá de la reestructuración y descentralización. In: Pereyra, M. et al. (Ed.). Globalización y descentralización de los sistemas 
¿Donde situar los esfuerzos de mejora?: política educativa, escuela y aula

educativos. Barcelona: Pomares-Corredor, 1996. p. 237-266. (Edição em português: Bolivar, A. O lugar da escola na política curricular actual: para além da reestructuração e da descentralização. In: SARMENTO, M. J. (Org.). Autonomia da escola: políticas e práticas. Porto: ASA, 1999. p. 157-190).

BOLÍVAR, A. Cómo mejorar los centros educativos. Madrid: Síntesis, 1999. (Edición portuguesa: Bolivar, A. Como melhorar as escolas?: estratégias e dinámicas de melhoria das práticas educativas. Porto: ASA, 2003.)

BOLÍVAR, A. Los educativos como organizaciones que aprenden: promesa y realidades. Madrid: La Muralla, 2000a.

BOLIVAR, A. Los centros escolares como comunidades: revisando la colegialidad. Revista Española de Pedagogía, Madrid, v. 58, n. 216, p. 253-274, mayo/agosto 2000b.

BOLÍVAR, A. Del aula al centro y ¿vuelta?: redimensionar el asesoramiento. In: Domingo, J. (Coord.). Asesoramiento al centro educativo: colaboración y cambio en la institución. Barcelona: Octaedro; Ediciones Universitarias de Barcelona, 2001. p. 51-68. Disponible en: <http://www.rinace.org> Acceso en: 25 ago. 2004.

BOLÍVAR, A. Si quiere mejorar las escuelas, preocúpese por capacitarlas: el papel del rendimiento de cuentas por estándares en la mejora. Profesorado, Revista de Currículum y Formación del Profesorado, Granada, v. 7, n. 1-2, p. 79-94, 2003. Disponible en: <http://www.ugr.es/ $\sim$ recfpro/Rev71ART4.pdf> Acceso en: 15 ago. 2004.

BOLÍVAR, A. El centro como unidad básica de acción educativa y mejora. In: Moreno, J.M. (Coord.). Organización y gestión de centros educativos. Madrid: UNED, 2004. p. 93-120.

CALHOUN, E.; JOYCE, B. 'Inside-out' and 'outside-in': learning from past and present school improvement paradigms. In: Hargreaves, A. et al. (Ed.). International handbook of educational change. Dordrecht: Kluwer, 1998. p. 1286-1298.

COHEN, D.K. Teaching practice: plus ça change. In: JACKsON, P. (Ed.). Contributing to educational change: perspectives on research and 
practice. Berkeley: McCutchan, 1989. p. 27-84. Disponible en: <http://ncrtl.msu.edu/http/ipapers/html/pdf/ip883.pdf >. Acceso en: 16 jun. 2004.

COHEN, D.K.; BALL, D.L. Instruction and innovation: reconsidering the story. Ann Arbor: University of Michigan, 2000. Disponible en: <http://www.sii.soe.umich.edu/links.html> Acceso en: 12 maio 2003.

COHEN, D.K.; HILL, H.C. Learning policy: when state education reform works. New Haven: Yale University, 2001.

COSTA, J.A. Proyectos educativos das escolas: um contributo para a sua (des)construção. Educação \& Sociedade, Campinas, v. 24, n. 85, p. 1319-1340, out. 2003.

DARLING-HAMMOND, L. El derecho de aprender: crear buenas escuelas para todos. Barcelona: Ariel, 2001.

DARLING-HAMMOND, L. et al. Building instructional quality: insideout, bottom-up, ant top-down perspectives on San Diego's school reform; paper presented for the Annual Meeting of the American Educational Research Association. Nueva Orleans, 2002.

EISNER, E. Estándares para las escuelas: ¿Una ayuda o un obstáculo?. In: EIsNer, E. La escuela que necesitamos: ensayos personales. Buenos Aires: Amorrortu, 2002. p. 248-264.

ELMORE, R.F. Teaching, learning, and school organization: principles of practice and the regularities of schooling. Educational Administration Quarterly, Columbus, v. 31, n. 3, p. 355-374, 1995 a.

ELMORE, R.F. Structural reform and educational practice. Educational Researcher, Washington, DC, v. 24, n. 9, p. 23-26, 1995 b.

ELMORE, R.F. The politics of education reform. Issues in Science and Technology, Washington, DC, v.14, n.1, 1997. Disponible en: <http:/ /www.nap.edu/issues/14.1/elmore.htm > Acceso en: 26 jun. 2004.

ELMORE, R.F. Building a new structure for school leadership. Washington, DC: Albert Shanker Institute, 2000. Disponible en: <www.shankerinstitute.org/> Acceso en: 24 jun. 2004. (Recogido también en ElMORE, R.F. School reform from the inside out: policy, practice, and performance. Cambridge, Mass.: Harvard University, 2004). 
¿Donde situar los esfuerzos de mejora?: política educativa, escuela y aula

ELMORE, R.E. Bridging the gap between standards and achievement: the imperative for professional development in education. Washington, DC: Albert Shanker Institute, 2002. Disponible en: <http://www.shankerinstitute.org> Acceso en: 5 ago. 2003. (Edición española: Bolivar, A. Salvar la brecha entre estándares y resultados: el imperativo para el desarrollo profesional en educación. Profesorado, Revista de Currículum y Formación del Profesorado, Granada, v. 7, n. 1-2, p.7-52, 2003). Disponible en: <http://www.ugr.es/ $\sim$ recfpro/Rev71ART1.pdf > Acceso en: 25 ago. 2004.

ELMORE, R.F.; PETERSON, P.L.; McCARTHEY, S.L. Restructuring in the classroom: teaching, learning, and school organization. San Francisco: Jossey-Bass, 1996. (Trad. y ed. del cap. 14. Enseñanza, aprendizaje y organización escolar. México, DF: Secretaría de Educación Pública, 2003).

ESCUDERO, J.M. Reforma, innovación y mejora. In: SAlvador Mata, F.; Rodríguez Diéguez, J.L.; Bolívar, A. (Dir.). Diccionario enciclopédico de didáctica. Málaga: Aljibe, 2004. v.2., p. 521-543.

ESCUDERO, J.M.; BOLÍVAR, A. Inovação e formação centrada na escola: uma perspectiva da realidade espanhola. In: Amiguinno, A.; CANÁRIO, R. (Org.). Escolas e mudança: o papel dos centros de formação. Lisboa: Educa, 1994. p. 97-155.

FUHRMAN, S.H.; ODDEN, A. Introduction: special section on school reform. Phi Delta Kappan, Bloomington, v. 83, n. 1, p. 59-61, 2001 .

FULLAN, M. Succesful school improvement: the implementation perspective and beyond. Buckingham: Open University, 1992.

FULLAN, M. The return of large-scale reform. Journal of Educational Change, v. 1, n. 1, p. 2-27, 2000a.

FULLAN, M. The three stories of educational reform. Phi Delta Kappan, Bloomington, v. 81 , n. 8, p. 581-584, 2000b

FULLAN, M. Las fuerzas del cambio: explorando las profundidades de la reforma educativa. Madrid: Akal, 2002a.

FULLAN, M. Los nuevos significados del cambio en la educación. Barcelona: Octaedro, 2002b. 
FULLAN, M. Liderar en una cultura de cambio. Barcelona: Octaedro, 2002c. (Edición portuguesa: Fullan, M. Liderar numa cultura de mudança. Porto: ASA, 2003).

FULLAN, M. Las fuerzas del cambio: la continuación. Madrid: Akal, 2004. GATHER THURLER, M. Inovar no interior da escola. Porto Alegre: ARTMED, 2001.

GOERTZ, M.E. Implementing standards-based reform: challenges for state policy. In: Duggan, T.; Holmes, M. (Ed.). Closing the gap: a report on the Wingspread Conference "Beyond the standards horce race. Washington, DC: Council for Basic Education, 2000. p. 65-82. Disponible en: <http://www.c-b-e.org/PDF/ClosingtheGap.pdf> Acceso en: 24 jun. 2003.

GONZÁLEZ, M.T. Los institutos de educación secundaria y los departamentos didácticos. Revista de Educación, Madrid, n. 333, p. 319-344, enero/abr. 2004.

HARGREAVES, A. Sustainability of educational change: the role of social geographies. Journal of Educational Change, v. 3, n. 3-4, p. 189-214, 2002.

HARGREAVES, A. Enseñar en la sociedad del conocimiento: la educación en la era de la inventiva. Barcelona: Octaedro, 2003. (Edición portuguesa: Hargreaves, A. O ensino na sociedade do conhecimento: a educação na era da insegurança. Porto: Porto, 2004).

HOPKINS, D. Tensions in and prospects for school improvement. In: Hargreaves, A. et al. (Ed.). International handbook of educational change. Dordrecht: Kluwer, 1998, p. 1035-1055.

HOPKINS, D.; LEVIN, B. Government policy and school development. School Leadership and Management, Oxfordshire, v. 20, n. 1, p. 15-30, 2000 .

HOPKINS, D.; REYNOLDS, D. The past, present and future os school improvement: toward the third age. British Educational Research Journal, Oxfordshire, v. 27, n. 4, p. 459-475, 2001.

KNAPP, M.S. et al. Leading for learning: sourcebook ; concepts and examples. Washington, DC: Center for the Study of Teaching and 
Policy, 2003. Disponible en: <http://depts.washington.edu/ctpmail/ PDFs/LforLSourcebook-02-03.pdf> Acceso en: 23 dez. 2003.

LEITHWOOD, K.; MENZIES, T. Forms and effects of school-based management: a review. Educational Policy, v. 12, n. 3, p. 325-346, 1998.

LOUIS, K.S.; TOOLE, J.; HARGREAVES, A. Rethinking school improvement. In: MurpHY, J.; LouIs, S.K. (Ed.). Handbook of research on educational administration. 2. ed. San Francisco: Jossey-Bass, 1999. p. 251276.

NEWMANN, F.; KING, M.B.; YOUNGS, P. Professional development that addresses school capacity: lessons from urban elementary schools. American Journal of Education, Chicago, v. 108, n. 4, p. 259-299, 2000. (Una versión de su presentación como paper en la AERA está disponible en: <http://www.wcer.wisc.edu/pdbo/grand-aje411.htm> Acceso en: 8 mar. 2004).

NEWMANN, F. et al. School instructional program coherence: benefits and challenges. Chicago: Consortium on Chicago School Research. 2001. Disponible en: <http://www.consortium-chicago.org/publications/p0d02.html> Acceso em: 8 mar. 2004.

SARASON, S.B. El predecible fracaso de la reforma educativa. Barcelona: Octaedro, 2003.

STOLL, L. Estrategias de mejora en los centros educativos (Entrevista con Louise Stoll). Organización y Gestión Educativa, n. 4, p. 26-30, 2002.

TALBERT, J.E.; McLAUGHLIN, M.W.; ROWAN, B. Understanding context effects on secondary school teaching. Teachers College Record, New York, v. 95, n. 1, p. 45-68, 1993.

WEILER, H.N. Enfoques comparados en descentralización educativa. In: Pereyra, M.A. et al. (Ed.). Globalización y descentralización de los sistemas educativos. Barcelona: Pomares-Corredor, 1996. p. 208-233. (Edición portuguesa: WeILER, H.N. Perspectivas comparadas em descentralização educativa. In: Sarmento, M. J. (Org.). Autonomia da escola: políticas e práticas. Porto: ASA, 1999. p. 157-190. 